\title{
Evolutionary sequence of expanding Hydrogen shells
}

\author{
M. Relaño ${ }^{1}$, J. E. Beckman ${ }^{2}$, O. Daigle ${ }^{3}$ and C. Carignan ${ }^{3}$ \\ ${ }^{1}$ Dpto. Física Teórica y del Cosmos, Universidad de Granada, Spain \\ email: mrelano@ugr.es \\ ${ }^{2}$ Instituto de Astrofísica de Canarias, C. Vía Láctea s/n, 38200, La Laguna, Tenerife, Spain \\ email: jeb@iac.es \\ ${ }^{3}$ Observatoire du mont Mégantic, LAE, Université de Montréal, C. P. 6128 succ. centre ville, \\ Montréal, Québec, Canada H3C 3J7 \\ email: odaigle@ASTRO.UMontreal.CA, carignan@ASTRO.UMontreal.CA
}

\begin{abstract}
Giant HI shells, with diameters of hundreds of parsecs and expansion velocities of 10$20 \mathrm{~km} \mathrm{~s}^{-1}$ are characteristic observed features of local gas rich galaxies. Although a predictable consequence of the impact of OB associations on the ISM doubts have been raised, as OB stars are not present in the centres of the majority of these shells. Here we combine our observations of expanding ionized shells in luminous $\mathrm{H}$ II regions with basic dynamical models to give support to the scenario in which OB associations do produce the HI shells.
\end{abstract}

Keywords. galaxies, ISM: H iI regions, kinematics and dynamics, bubbles

From Fabry-Pérot data cubes in $\mathrm{H} \alpha$ of four late-type spiral galaxies: NGC 1530, NGC 3359, NGC 5194 and NGC 6951, we extract emission line profiles of the most luminous H II regions. Symmetrically placed about the central dominant peak we find two peaks of low intensity but high velocity in the profiles of all regions with sufficient S:N. We interpret these as clear evidence for supersonic expanding shells within the regions, and use them to quantify the masses and kinetic energies of these shells (cf. Relaño \& Beckman 2005). Using a basic model in which energy is supplied first by stellar winds and then by supernovae (Dyson 1981) we extrapolate the properties of these shells in time, finding results in good overall agreement with the observed properties of the HI shells, in mass and velocity ranges, after times of a few $10^{7} \mathrm{yr}$. If the formation of the OB stars occurs on timescales notably shorter than this, these stars will no longer be found at the centres of the HI shells, in agreement with observations reported by Hatzidimitriou et al. (2005) in the SMC.

\section{References}

Relaño, M. \& Beckman, J. E. 2005, A\& A 430, 911

Dyson, J.E. 1981 in: F. D. Kahn (ed.), Investigating the Universe (Dordrecht: Reidel), p. 125

Hatzidimitriou, D., Stanimirovic, S., Maragoudaki, F., Staveley-Smith, L., Dapergolas, A., \& Bratsolis, E. 2005, MNRAS 360, 1171 